\title{
Feasibility of using an led-probe in third- space endoscopy: a clinical study
}

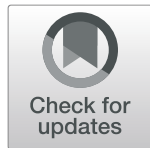

\author{
Oscar Víctor Hernández Mondragón*, Raúl Zamarripa Mottú, Omar Solórzano Pineda, \\ Raúl Alberto Gutierrez Aguilar and Luís Fernando García Contreras
}

\begin{abstract}
Background: Third-space endoscopy is a novel, safe, and effective method for treating different gastrointestinal conditions. However, several failed endoscopic procedures are attributed to incomplete myotomy. Lighting devices are used to prevent organic injuries. We aimed to investigate the feasibility of using a hand-made LED-probe (LP) in third-space procedures.

Methods: This prospective study was conducted in a tertiary-care center in Mexico between December 2016 and January 2019. We included peroral endoscopic myotomy (POEM) and gastric peroral endoscopic myotomy(G-POEM) procedures. Pseudoachalasia, peptic ulcer, normal gastric emptying scintigraphy (GES) and prepyloric tumors were excluded. LP was used to guide or confirm procedures. Clinical and procedural characteristics were recorded and analyzed.
\end{abstract}

Results: Seventy third-space procedures were included (42POEM,28G-POEM), with an average patient age of $46.7 \pm$ 14.3 and $43.7 \pm 10.1$ years, respectively. For the POEM and G-POEM groups, respectively, 18/42(42.9\%) and 13/ 28(46.7\%) patients were males; median procedure times were 50 (interquartile range [IQR]: 38-71) and 60(IQR: 4877) min, median LP placement times were 5(IQR: 4-6) and 6(IQR: 5-7) min, mild adverse events occurred in 4(9.4\%) and $4(14.2 \%)$ of cases, and clinical success at 6 months occurred in 100 and $85.7 \%$ of cases. Integrated relaxation pressure (IRP) improved from $27.3 \pm 10.8$ to $9.5 \pm 4.1 \mathrm{mmHg}(p<0.001)$; retention percentage at $4 \mathrm{~h}$ also improved. LP was successfully placed and adequate myotomy confirmed including 14.2 and $17.8 \%$ of POEM and G-POEM difficult patients.

Conclusions: Using an LP is promising and allows guiding during third-space procedures either for submucosal tunnel creation or myotomy confirmation, with excellent safety and efficacy in clinical practice.

Keywords: Third-space endoscopy, LED-probe, Peroral endoscopic myotomy, Gastric peroral endoscopic myotomy

\section{Background}

Third-space endoscopy has emerged as a novel and effective method for the treatment of different gastrointestinal disorders such as achalasia and refractory gastroparesis [1-4]. However, difficult or incomplete myotomy has been identified in peroral endoscopy myotomy (POEM) and gastricperoral endoscopy myotomy (G-POEM) as a result of

\footnotetext{
* Correspondence: mondragonmd@yahoo.co.uk

Division of Endoscopy, Specialties Hospital, National Medical Center Century XXI, Cuauhtémoc Avenue 330, 06700 México City, Mexico
}

different factors such as end-stage disease or previously treated cases (due to high submucosal fibrosis) [1, 5], inability to identify the esophagogastric junction (EGJ) throughout the submucosal tunnel during POEM [6-8] or the pyloric muscle ring (PMR) during G-POEM [9], lack of third-space endoscopy experience $[2,3,6]$, and certain anatomical factors (megaesophagus, sigmoid-type) $[4,9,10]$. These factors are associated with a failed procedure. Different techniques, such as fluoroscopy $[9,11]$ or double endoscopy $[12,13]$ have been proposed to overcome these problems and guide

(c) The Author(s). 2020 Open Access This article is licensed under a Creative Commons Attribution 4.0 International License, which permits use, sharing, adaptation, distribution and reproduction in any medium or format, as long as you give appropriate credit to the original author(s) and the source, provide a link to the Creative Commons licence, and indicate if changes were made. The images or other third party material in this article are included in the article's Creative Commons licence, unless indicated otherwise in a credit line to the material. If material is not included in the article's Creative Commons licence and your intended use is not permitted by statutory regulation or exceeds the permitted use, you will need to obtain permission directly from the copyright holder. To view a copy of this licence, visit http://creativecommons.org/licenses/by/4.0/ The Creative Commons Public Domain Dedication waiver (http://creativecommons.org/publicdomain/zero/1.0/) applies to the data made available in this article, unless otherwise stated in a credit line to the data. 
an appropriate tunnel creation and myotomy; however, low availability and high costs limit their use.

Lighting devices for surgical guidance have been investigated for years with good results. In laparoscopic colorectal and gynecological surgeries, the use of lighted ureteral stents has successfully prevented iatrogenic injuries $[14$, 15]. In ophthalmologic procedures, light-emitting diode (LED) technology is used for vitrectomy, with the advantage of no thermal or photodynamical harm; this provides better illumination than other light sources $[16,17]$. In third-space endoscopy, the use of a dedicated LED-probe (LP) that could be inserted into the submucosal tunnel (cornerstone of the procedure) $[1,2,8]$ could be used to guide the direction during the procedure or confirm a correct myotomy when combined with the endoscope, before closing the entry site. Use of an LP could be an excellent alternative during third-space endoscopic procedures. The aim of this study was to evaluate the feasibility of using of LP in clinical practice, especially during POEM or GPOEM third-space procedures.

\section{Methods}

\section{Study design and ethical considerations}

This prospective study was conducted in a tertiary care center in Mexico City, Mexico between December, 2016 and January, 2019. We included patients between the ages of 18 and 90 years with naïve or previously-treated achalasia or severe refractory gastroparesis and who were treated with POEM or G-POEM, respectively, with 6 months of follow-up. Patients with pseudoachalasia were excluded from the POEM procedure. Active peptic ulcer disease, normal gastric emptying scintigraphy (GES) and prepyloric tumor lesions were excluded from G-POEM. Patients with severe clinical conditions that could be contraindications to any of these procedures were also excluded (severe chronic obstructive pulmonary disease, recent myocardial infarction). This protocol was approved by the Local Ethics Committee (R-20163601-192; registration number: 2016-CMN675). Informed consent was obtained from all patients.

\section{Poem}

\section{Patients}

The diagnosis of achalasia was based on the Chicago Classification [18], using high resolution manometry (HRM). Upper gastrointestinal (GI) endoscopy, computed tomography (CT) scanning, a timed barium esophagogram (TBE) and the Chagas disease test were performed. Esophageal classification was evaluated according to Rezende's classification [19]; the Eckardt score was used for clinical evaluation [20].

\section{Procedure}

The esophagus was cleaned $24 \mathrm{~h}$ before the POEM procedure and antibiotic prophylaxis with third-generation cephalosporines or quinolones was administered. All procedures were performed under general anesthesia. A 9. 8-mm outer diameter with a 2. 8-mm working channel endoscope was used (EG590WR; Fujinon, Tokyo, Japan), along with a transparent cap model (DH-28GR, Fujinon). An electrosurgical unit (ERBE VIO-200D, Tübingen, Germany), and an I-type hybrid knife (ERBE, Tübingen, Germany) were also used. Closure was performed using hemoclips (Boston Scientific, Natick, Massachusetts, USA).

The LP system was created using a sterile polyurethane non-weighted $127 \mathrm{~cm} \times 20 \mathrm{Fr}$ nasogastric feeding tube (AMA Proveduria, Mexico City, Mexico) that was cut at the middle. A thin strip of $150 \mathrm{cms}$ long of an ultrabright slim LED strip light system of $1 / 8$ in $[3.5 \mathrm{~mm}]$ in diameter, with a capability of $112 \mathrm{~lm} / \mathrm{m}$ (FlexfireLEDs, Costa Mesa, California, USA), was inserted throughout this catheter and attached to it with one $127 \mathrm{~cm}$ long strip of Scotch black tape (3 M, St. Paul, Minnesota, USA). This LP was coupled to a power supply system of $12 \mathrm{~V}$ that used conventional alkaline AA batteries (Duracell, Bethel, Connecticut, USA). After LP creation, it was cleaned with soapy water, rinsed and then dried. Finally, we used an antiseptic wipe (SoluPrep $^{\text {tw }}, 3$ M, St. Paul, Minnesota, USA), which has $2 \%$ w/v chlorhexidine gluconate and $70 \% \mathrm{v} / \mathrm{v}$ isopropyl alcohol, in order to clean the catheter before use (video 1).

The POEM technique was based on Inoue's technique [21]. The technique was performed using the following procedure: (1), initially, the esophagus was cleaned if necessary; (2), injection and incision were performed with a mixture of saline solution and $0.5 \%$ methylene blue being injected $13-15 \mathrm{~cm}$ above the EGJ ( $20 \mathrm{~cm}$ for type III patients) and a $12-15 \mathrm{~mm}$ longitudinal incision being made for anterior (naïve patients) or posterior (previously-treated patients) approaches; (3), the submucosal tunnel was created, with the tunnel being created up to $3 \mathrm{~cm}$ below the EGJ; (4), a full-thickness myotomy was performed in all patients; (5), the LP was placed through the mouth; once it passed the cricopharyngeal muscle, it was grasped using biopsy forceps under endoscopic visualization and inserted up to the end of the submucosal tunnel; (6), intraluminal revision of the POEM procedure was performed and EGJ was reviewed in retroflexion with the LP on, in order to confirm that submucosal tunnel reached gastric space and an adequate myotomy was performed. If LP on was not observed, submucosal tunnel and myotomy were considered as insufficient and they were corrected as necessary until LP on was observed. Submucosal tunnel was reviewed again to rule out adverse events secondary to LP or endoscope at this level; (7), closure with clips at the entry site was performed (Fig. 1).

\section{Follow-up}

Twenty-four hours after POEM, upper GI endoscopy and upper GI series were performed to rule out adverse 


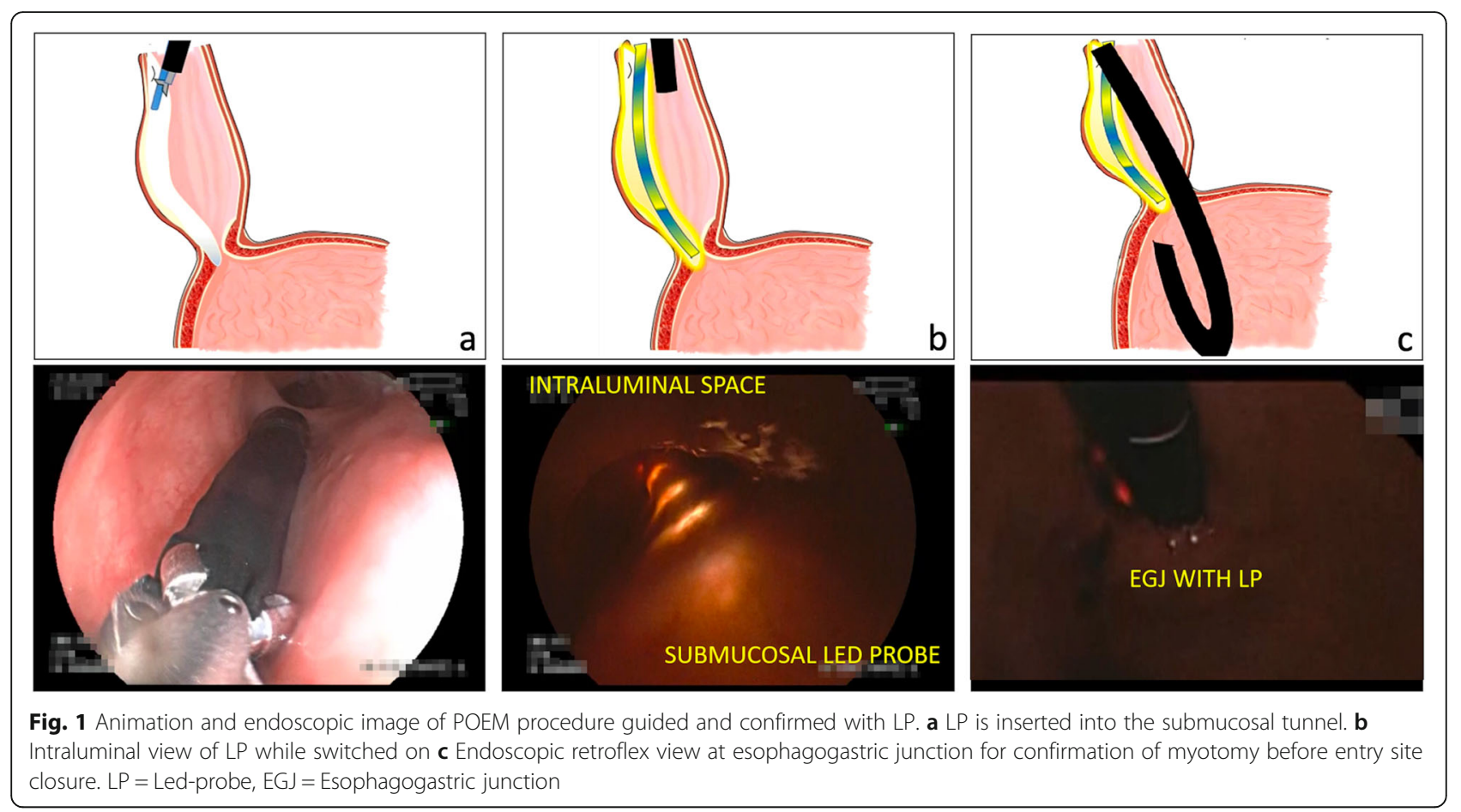

events. Antibiotics were continued by intravenous and then oral route for 7 days. A proton pump inhibitor (PPI) was administered for 4 weeks. Diet progressed from a liquid diet up to a normal one over the following 3 weeks. Follow-up continued for 6 months, with HRM, GI endoscopy, pHmetry, TBE, reflux questionnaires, and Eckardt scores evaluated at 3 and 6 months. Success was defined as an integrated relaxation pressure (IRP) $<15$ $\mathrm{mmHg}$, Eckardt score $<3$, and TBE demonstrating adequate passage of contrast $(\geq 80 \%$ at $5 \mathrm{~min}$ ) 3 and 6 months postoperatively.

\section{G-poem}

\section{Patients}

Gastroparesis was diagnosed based on clinical evaluations and scintigraphy. Severe refractory gastroparesis was based on the presence of delayed gastric emptyingrelated symptoms, including nausea, retching, vomiting, abdominal pain, post-prandial fullness, early satiety and/ or bloating. Patients who had failure or recurrence after receiving optimal pharmacological therapies and a Gastroparesis Cardinal Symptom Index (GCSI) score $>2.3$ (score that has been validated as severe when greater than 2.3) [22] with a retention percentage at $4 \mathrm{~h}(\mathrm{RP} 4 \mathrm{H})$ in GES > 10\% and a mean half emptying time (MHET) $>150 \mathrm{~min}$, were also diagnosed with severe refractory gastroparesis. Efficacy was evaluated based on a reduction in the self-reported gastroparetic symptoms of the patients; the absence of recurrent hospitalizations and the proportion of patients with a decrease in GCSI score $<2.3, \mathrm{RP} 4 \mathrm{H}<10 \%$ and $\mathrm{MHET}<150 \mathrm{~min}$. Adverse events in POEM and G-POEM were graded according to the American Society for Gastrointestinal Endoscopy Lexicon [23].

\section{Procedure}

Procedures were performed in the endoscopic unit under general anesthesia. Forty-eight hours before the procedure, all patients were administered a liquid diet and antibiotic prophylaxis with third-generation cephalosporines or quinolones. Endoscopic instruments were the same as in POEM patients, including the LP.

The G-POEM procedure was based on Khashab's technique [24]. The technique was performed based on the following protocol: (1), revision and cleaning of the stomach were performed; (2), injection and incision were performed $5 \mathrm{~cm}$ before the pylorus for submucosal bleb creation using a combination of saline solution with $0.5 \%$ methylene blue. A longitudinal $1.5 \mathrm{~cm}$ incision was also performed; (3), a submucosal tunnel was performed in pyloric direction until PMR was identified. If submucosal tunnel orientation was lost during this step, LP was used and was inserted throughout the mouth, grasped with biopsy forceps and placed intraluminally into the duodenal bulb in order to guide submucosal tunnel creation and PMR identification; (4), myotomy of the circular and longitudinal muscular layers of the pylorus and the antrum was performed; the incision was 3$4 \mathrm{~cm}$ in length and deep up to the serosa. LP was used for myotomy confirmation in all cases. Before closure 
was performed, submucosal tunnel was reviewed endoscopically to rule out complications at this level; (5), the incision was closed using the over the scope clips (OTSC) type A (OVESCO Endoscopy, AG, Tübingen, Germany) or hemoclips (Fig. 2).

\section{Follow-up}

Patients were admitted to the hospital after the procedure and given intravenous antibiotics, and then changed to oral route, in order to complete 7 days. An upper GI series and upper endoscopy were performed at $24 \mathrm{~h}$ to rule out adverse events. Diet progressed from a liquid diet to a normal diet over the next week. PPI was administered for 4 weeks. Both GCSI and GES were performed 3 and 6 months postoperatively.

\section{Statistical analyses}

Sample size was calculated based on an assumption that there would be at least $90 \%$ of efficacy in the completion of third-space procedures when LP is used, with a significance level of 0.05 (type I error of 5\%) and a beta of 0.20 (type II error of 20\%). Using an online statisticallyvalidated program for sample size calculation (EpiInfo, USA), we calculated a minimum of 25 patients for POEM and G-POEM procedures. Quantitative data were expressed as mean (standard deviation [SD]) or median with interquartile range (IQR); qualitative data were expressed as frequencies and percentages. Bivariate comparisons were done using the Friedman test, chi-squared test and a one-way analysis of variance, as appropriate.
$P<0.05$ was considered to be statistically significant. SPSS v.23.0 (IBM, Chicago, Illinois, USA) was used for all statistical analyses.

\section{Results}

There were 70 third-space procedures performed between December 2016 and January 2019 (42 POEM and 28 G-POEM).

\section{Poem \\ Baseline characteristics}

Forty-two patients were included with a mean age of $46.7 \pm 14.3$ years and $18(42.9 \%)$ were male. Esophagus type grade II (16 [38\%]) and achalasia subtype II (20 [47.6\%]) were the most common. Thirty-two (76.2\%) were naïve, while 10 (23.8\%) had been previously treated, (60\% had undergone LHM, 20\% botulinum toxin injection, and $20 \%$ pneumatic dilation). No Chagas disease was found.

\section{Procedure}

The median total POEM time was 50 min (IQR 38-71). The mean tunnel and myotomy lengths were $12.9 \pm 3.6$ $\mathrm{cm}$ and $10.5 \pm 3.1 \mathrm{~cm}$, respectively. The most common mild adverse event was minor bleeding in 2 cases (4.7\%), and the median length of stay (LOS) was 3 days (IQR 1-4).

\section{Efficacy}

Clinical response was observed in all cases at the 6month follow-up. The Eckardt score decreased from 9 to
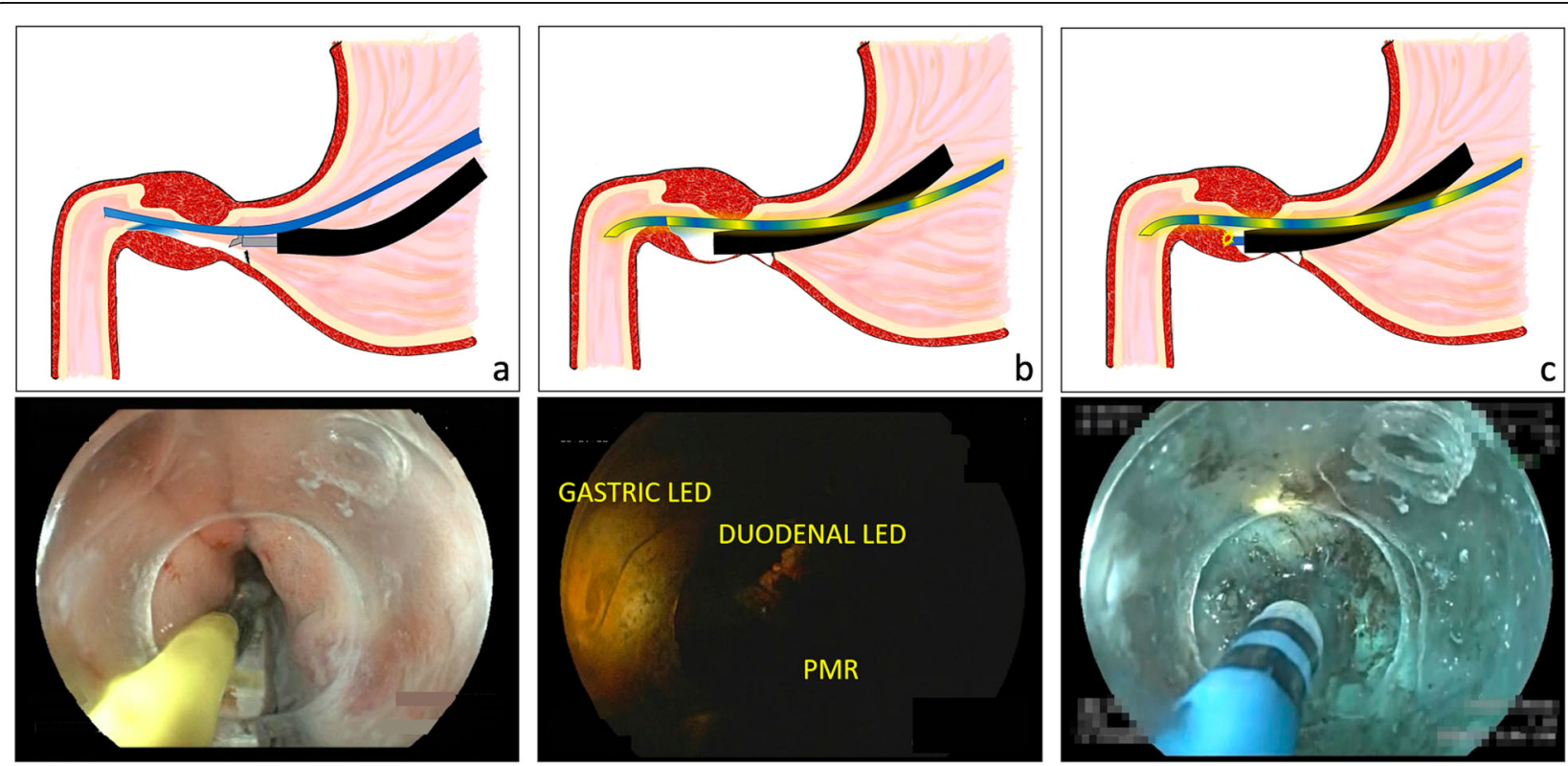

Fig. 2 Animation and endoscopic image of G-POEM procedure guided and confirmed with LP. a LP is inserted intraluminally into the duodenal bulb. b Endoscopic view of LP throughout the submucosal space. Gastric and duodenal LED lights are observed with PMR at the bottom. $\mathbf{c}$ Myotomy is performed with LP guidance 
1 at the 3-month follow-up $(P<0.001)$ and did not change at the 6-month follow-up $(P<0.001)$. The IRP decreased from $27.3 \pm 10.8 \mathrm{mmHg}$ to $9.8 \pm 3.8 \mathrm{mmHg}$ at the 3 month follow-up $(P<0.001)$ and $9.5 \pm 4.1 \mathrm{mmHg}$ at the 6month follow-up $(P<0.001)$; TBE showed emptying of $<$ $50 \%$ in $100 \%$ of patients to emptying of $>50 \%$ in $100 \%$ $(p>0.001)$ after 6 months. Furthermore, 57\% presented positive pHmetry, $15 \%$ had esophagitis and $12 \%$ had clinical symptoms of reflux disease (Table 1).

\section{G-poem}

\section{Baseline characteristics}

Twenty-eight patients with a mean age of $43.7 \pm 10.1$ years were included and 13 were male (46.4\%). The most common etiology was diabetes in $12(42.9 \%)$ and the mean duration of disease before G-POEM was $22.2 \pm 5.5$ months. The most predominant symptoms and previous therapy were: nausea and vomiting in 15 (53.5\%), and medical therapy in $22(78.7 \%)$, respectively. The median number of hospitalizations preoperatively was 2 (IQR 2-5).

\section{Procedure}

The median total G-POEM time was 60 min (IQR 4877). The mean tunnel and myotomy lengths were $5.2 \pm$ $0.96 \mathrm{~cm}$ and $3.2 \pm 0.82 \mathrm{~cm}$, respectively. The most common mild adverse event was capnoperitoneum in 2 patients (7.1\%); it required abdominal decompression with a Veress needle and 1 mucosal tear $24 \mathrm{~h}$ after procedure and was solve endoscopically with clips. The median LOS was 2 days (IQR 1-6).

\section{Efficacy}

The GSCI score decreased from $3.5 \pm 0.64$ points to $1.8 \pm 0.61$ after 3 months $(P<0.001)$, and $1.2 \pm 0.43$ after 6 months $(P<0.001)$. GES test showed a decrease in RP4H from $35.3 \pm 11.6$ to $11.1 \pm 4.2$ after 3 months $(P<$ $0.001)$, and $9.3 \pm 3.2$ after 6 months $(P<0.001)$. The halfemptying time improved from $260.2 \pm 66.9 \mathrm{~min}$ to $165.9 \pm 31.2$ min after 3 months $(P<0.001)$, and $152.7 \pm$ 23.1 min after 6 months $(P<0.001)$. Clinical response was observed in 24 patients $(85.7 \%)$ at the 6-month evaluation. Resolution of the predominant symptoms were as follows: resolved in $16(57.1 \%), 18(64.3 \%)$ and 9 (32.1\%); improved in 10 (35.8\%), $5(17.8 \%)$ and 11 (39.2\%); not changed in 1 (3.6\%), $3(17.8 \%)$ and 7 (25\%) and worsened in 1 (3.6\%), $2(7.2 \%)$ and 1 (3.6\%), for nausea/vomiting, abdominal pain and gastric fullness, respectively. GES was normalized in 17 (60.7\%) and partially improved in $8(28.5 \%)$ of patients at the 6-month evaluation (Table 2).

\section{LED probe}

The median placement time for POEM and G-POEM were 5 (IQR 4-6) and 6 (IQR 5-7) min, respectively. All probes were successfully placed without adverse events and no adverse events or technical failures were observed after procedures secondary to their use. There was no damage to the submucosal space or at the intraluminal mucosal level in both POEM and G-POEM cases, and we didn't have any associated infections, neither when LP was placed in the mediastinal space at EGJ level in POEM cases. In general, LP use helped to adequately complete POEM and G-POEM in 11/70 $(15.7 \%)$ of cases. After initial classic POEM procedure, LP on was placed and not observed in 6/42(14.2\%) of cases. Therefore, submucosal tunnel and myotomy were extended up to an adequate LP confirmation. In POEM cases, placement of LP in posterior approach was relatively easier than anterior approach, but without significant differences in placement times (4 vs $6 \mathrm{~min} ; P=0.2$ ). In G-POEM cases, inadequate submucosal direction was found in 5/28(17.8\%) of cases and LP use helped to correct it and confirm myotomy of the PMR in all cases. (video 2).

\section{Discussion}

In this study we evaluated the feasibility of using a new device, the LP, and confirmed its safe and effective use in clinical practice when performing third-space procedures (POEM and G-POEM).

Third-space endoscopy was first described by Sumiyama et al. [25]; it was first used in animals in 2007, and then used in humans. It is based on the creation of a submucosal tunnel to perform surgical procedures with confirmed safety and efficacy. It transforms the concept of endoluminal endoscopy to intramural, making many diseases that previously would have been treated by open or laparoscopic surgery endoscopically curable $[1,4]$.

The lumen is considered as the first space, while the peritoneum is considered the second space and the intentionally-created tunnel is the third space (space between the mucosa and muscularis propria) [2-4]. Different disorders have been addressed by this technique, including Zenker's diverticulum, myotomy for achalasia, gastroparesis, Hirschsprung's disease, removal of tumors arising from the muscularis propria and beyond, and stricture treatment $[1,2,8]$. Among them, achalasia and gastroparesis are the most prevalent diseases treated by this technique. However, "difficult", "incomplete", or even "not possible" tunnel creation or myotomy have been described and are associated with certain complexity factors such as end-stage disease (POEM) [5], previouslytreated cases (POEM and G-POEM) $[4,8,24]$, inability to identify EGJ (POEM) [6-8], PMR (G-POEM) [9], high submucosal fibrosis (POEM and G-POEM) [2-4, 6-8], lack of experience (POEM and G-POEM) [2, 3, 6],or anatomical factors (POEM) $[1,4,9,10]$. These factors result in a failed procedure, even if the endoscopist believes that 
Table 1 Characteristics of the 42 POEM procedures performed with LED probe

\begin{tabular}{|c|c|c|c|c|}
\hline $\begin{array}{l}\text { Patients } \\
N=42\end{array}$ & Value & & & \\
\hline Age, mean $(S D)$, years & $46.7 \pm 14.3$ & & & \\
\hline Sex,male,n (\%) & $18(42.9 \%)$ & & & \\
\hline \multicolumn{5}{|l|}{ Type of esophagus, n (\%) } \\
\hline • Normal & $2(5 \%)$ & & & \\
\hline - Grade I & $8(19 \%)$ & & & \\
\hline - Grade II & $16(38 \%)$ & & & \\
\hline - Grade III & $8(19 \%)$ & & & \\
\hline - Grade IV & $8(19 \%)$ & & & \\
\hline \multicolumn{5}{|l|}{ Previous treatments, n (\%) } \\
\hline - Treatment naïve & $32(76.2 \%)$ & & & \\
\hline - 'Previously treated & $10(23.8 \%)$ & & & \\
\hline$\cdot+$ Post-LHM & $6(60 \%)$ & & & \\
\hline •+Botulinum toxin injection & $2(20 \%)$ & & & \\
\hline •+Pneumatic dilation & $2(20 \%)$ & & & \\
\hline \multicolumn{5}{|l|}{ Achalasia subtype, n (\%) } \\
\hline • Type I & $11(26.2 \%)$ & & & \\
\hline • Type II & $20(47.6 \%)$ & & & \\
\hline • Type III & $11(26.2 \%)$ & & & \\
\hline \multicolumn{5}{|l|}{ Procedure } \\
\hline Tunnel length, mean (SD), cm & $12.9 \pm 3.6$ & & & \\
\hline Myotomy length, mean (SD) cm & $10.5 \pm 3.1$ & & & \\
\hline LP placement time, median (IQR), min & $5(4-6)$ & & & \\
\hline $\begin{array}{l}\text { Patients with inadequate myotomy } \\
\text { after initial classic POEM that benefited } \\
\text { from LP use (difficult cases), n (\%) }\end{array}$ & $6(14.2 \%)$ & & & \\
\hline Total POEM time, median (IQR), min & $50(38-71)$ & & & \\
\hline Adverse Events, n (\%) & $4(9.4 \%)$ & & & \\
\hline • Minor bleeding & $2(4.7 \%)$ & & & \\
\hline • Pneumoperitoneum & $2(4.7 \%)$ & & & \\
\hline POEM outcomes & PRE-POEM & POST-POEM 3 m & POST-POEM $6 \mathrm{~m}$ & $P$ value \\
\hline Eckardt score, median (IQR), points & $9(6-12)$ & $1(0-3)$ & $1(0-3)$ & $<0.001^{1}$ \\
\hline IRP pressure, mean (SD), mmHg & $27.3 \pm 10.8$ & $9.8 \pm 3.8$ & $9.5 \pm 4.1$ & $<0.001^{2}$ \\
\hline TBE & & & & $<0.001^{3}$ \\
\hline$\cdot<50 \%$ & $100 \%$ & $0 \%$ & $0 \%$ & \\
\hline$\cdot 50-80 \%$ & $0 \%$ & $14 \%$ & $9.5 \%$ & \\
\hline$\cdot>80 \%$ & $0 \%$ & $86 \%$ & $90.5 \%$ & \\
\hline
\end{tabular}

SD standard deviation, IQR interquartile range, POEM peroral endoscopic myotomy, LP led-probe, LHM laparoscopic Heller myotomy

${ }^{1}$ Friedman test

${ }^{2}$ ANOVA test

${ }^{3} X^{2}$ test

the procedure was successful $[1,3]$. If the tunnel is too short, the procedure is ineffective; if the length of the myotomy is too long, there is a higher risk of adverse events, including perforation or bleeding [3, 12]. Currently, there are several endoscopic landmarks, such as palisading vessels at the EGJ and the circular bundle of
LES fibers in POEM (difficult use and inaccurate), or the continuous insertion and extraction of the endoscope from the tunnel, that are used to identify the PMR, which is technically challenging. A second endoscope (POEM and G-POEM) $[12,13]$ and fluoroscopy (G-POEM) $[9,11]$ are used in an attempt to overcome these problems; 
Table 2 Characteristics of the 28 G-POEM procedures performed with LED probe

\begin{tabular}{|c|c|c|c|c|}
\hline $\begin{array}{l}\text { Patients } \\
N=28\end{array}$ & Value & & & \\
\hline Age, mean (SD), years & $43.7 \pm 10.1$ & & & \\
\hline Sex, male, n (\%) & $13(46.4)$ & & & \\
\hline \multicolumn{5}{|l|}{ Etiology, n (\%) } \\
\hline - Diabetic & $12(42.9 \%)$ & & & \\
\hline • Idiopathic & $11(39.2 \%)$ & & & \\
\hline • Postsurgical & $5(17.9 \%)$ & & & \\
\hline Duration of disease before G-POEM, mean (SD), months & $22.2 \pm 5.5$ & & & \\
\hline \multicolumn{5}{|l|}{ Predominant symptoms, n (\%) } \\
\hline - Nausea/vomiting & $15(53.5 \%)$ & & & \\
\hline - Abdominal pain & $8(28.6 \%)$ & & & \\
\hline - Gastric fullness & $5(17.9 \%)$ & & & \\
\hline \multicolumn{5}{|l|}{ Previous therapy, n (\%) } \\
\hline - Medical treatment & $22(78.7 \%)$ & & & \\
\hline - Botulinum toxin injection & $5(17.8 \%)$ & & & \\
\hline • Transpyloric stenting & $1(3.5 \%)$ & & & \\
\hline \multicolumn{5}{|l|}{ Procedure } \\
\hline Tunnel length, mean (SD), cm & $5.2 \pm 0.96$ & & & \\
\hline Myotomy length, mean (SD), cm & $3.2 \pm 0.82$ & & & \\
\hline LP placement time, median (IQR), min & $6(5-7)$ & & & \\
\hline $\begin{array}{l}\text { Patients with inadequate submucosal } \\
\text { tunnel direction after initial classic } \\
\text { G-POEM procedure that benefited } \\
\text { from LP use, } \mathrm{n}(\%)\end{array}$ & $5(17.8 \%)$ & & & \\
\hline Total G-POEM time, median (IQR), min & $60(48-77)$ & & & \\
\hline Adverse Events, n (\%) & $4(14.2 \%)$ & & & \\
\hline • Capnoperitoneum & $2(7.1 \%)$ & & & \\
\hline - Mucosal tear & $1(3.5 \%)$ & & & \\
\hline - Prepyloric ulcer & $1(3.5 \%)$ & & & \\
\hline G-POEM outcomes & PRE-GPOEM & POST-GPOEM 3 m & POST-GPOEM 6 m & $P$ value \\
\hline GSCl score, mean (SD), points & $3.5 \pm 0.64$ & $1.8 \pm 0.61$ & $1.2 \pm 0.43$ & $<0.001^{1}$ \\
\hline $\mathrm{RP} 4 \mathrm{H}$, mean (SD), percentage & $35.3 \pm 11.6$ & $11.1 \pm 4.2$ & $9.3 \pm 3.2$ & $<0.001^{1}$ \\
\hline MHET, mean (SD), minutes & $260.2 \pm 66.9$ & $165.9 \pm 31.2$ & $152.7 \pm 23.1$ & $<0.001^{1}$ \\
\hline
\end{tabular}

$S D$ standard deviation, IQR interquartile range, G-POEM gastric peroral endoscopic myotomy, LP led-probe, GSCl gastroparesis cardinal symptoms index, RPH4 retention percentage $4 \mathrm{~h}$, MHET mean half emptying time

1 ANOVA test

however, these methods are costly or are unavailable. Therefore, we decided to explore a new alternative to overcome these problems when performing third-space endoscopy.

The use of lighting devices has been explored in medicine for years. In colorectal and gynecological surgeries, for example, iatrogenic ureteric injury is a serious complication with a variable incidence between $0.7-10 \%$ [14]. The identification of ureters is challenging and the optional double J stent placement is invasive and associated with serious adverse events [15];however, the use of fluorescence and lighted ureteral stents has overcome these problems. LED technology was invented in 1907 by H. J. Round but was commercially available in 1962 in electrical components. Modern LED technology with more practicality was used after 2010 [17]; uses in medicine are confirmed in ophthalmologic procedures where improved illumination for vitrectomy has been observed [16].LED has advantages over incandescent light sources. It provides lower energy consumption, a longer lifetime, smaller size, faster switching, a better spectrum of light and intensity (emitting more lumens per watt compared 
with light bulbs), and cool light that radiates minimal heat. It is safe because mercury or other hazardous metals are not contained within it $[16,17]$.

Because of safety and efficiency, we decided to use a white LED-probe and orally insert it into a conventional $127 \mathrm{~cm} \times 20 \mathrm{Fr}$ nasogastric feeding tube. We spent between 10 to $15 \mathrm{~min}$ for LP building and disinfection process. Excellent visualization was obtained with the $112 \mathrm{~lm} / \mathrm{m}$ of the probe; this was enough to be visualized under the submucosal tunnel or over the intraluminal space when inserted into the tunnel. We didn't have technical problems during assembly or during procedures, neither when insertion into the patient was performed or after LP was used and procedures were finished. Therefore, based on these results, we confirmed the safety and efficacy of this device, that had a median insertion time of 5 min (4-6) for POEM and 6 (5-7) for G-POEM, without compromising total procedural times, and being similar to those observed in previous studies $[5-7,10,11,21,24]$.

POEM was performed as described by other groups $[5,6,21]$, with similar demographic characteristics. However, in our cohort $23.8 \%$ were previously treated and $38 \%$ grade III and IV. These are the subgroups theoretically more difficult to treat; therefore, with the greatest benefit if LP is used. Nonetheless, POEM was completed in $100 \%$ of cases and the mean myotomy length was $10.5 \pm 3.1 \mathrm{~cm}$, which is similar to the length of $9.4 \pm 3.1 \mathrm{~cm}$ obtained in other studies [6]. Grimes et al. [13] compared double-scope vs conventional POEM in a clinical trial that included $50 \mathrm{pa}-$ tients per group. No differences in technical (98\% vs $100 \%$ ) or clinical success (93\% vs 97\%) was found, but with a $34 \%$ longer myotomy and 17 min increase in procedural times for double-scope group. In our study, the LP was $6 \mathrm{~mm}$ in diameter, which is similar to the length of the neonatal endoscope used in Grimes' study. However, our LP system was advantageous in terms of cost (10 dls per LP), and placement time ( $5 \mathrm{~min}$ vs $17 \mathrm{~min}$ ). We confirmed an adequate myotomy in all cases including 6 patients (14.2\%) who were considered as difficult (4 grade IV, 1 grade III and 1 pneumatic dilation), in whom classic POEM didn't complete myotomy and who benefited from the LP use, avoiding potential adverse events or risk of incomplete procedure. Additionally, no other endoscopy tower was needed (saving costs and space in the endoscopy room).. In 2019, Grimes et al. published the follow-up of the cohort of double-scope vs conventional POEM, with a median of 3 years. They found no differences in clinical outcomes between groups $(83 \%$ vs $80 \% ; P=1.0)$, without differences in reflux disease incidence, but more cases with grade B esophagitis were presented in treatment group (25\% vs $4 \% ; P=0.049$ ); they hypothesize that this is because a longer myotomy is performed in them $(1.6 \pm 1.2 \mathrm{cms})$ [26] . In our cases, the LP allowed performing an adequate EGJ myotomy and, at 6-month evaluation, clinical outcomes and the occurrence of reflux disease was similar to those of other studies $[5-8,12,13,21]$. This suggests that the adequate confirmation of EGJ myotomy is the most important step in POEM procedures, regardless of whether an external device is used or not. Confirmation, which should be performed in all cases, mostly in earlyexperienced endoscopists in POEM procedure, represents LP as an excellent alternative for this purpose.

We performed the G-POEM and LP placement in all cases. Demographic characteristics were similar to other groups $[8-11,24]$. The pylorus was previously manipulated in $21.4 \%$ of cases (botulinum toxin injection and transpyloric stent), potentially difficult cases. However, median G-POEM time $(60 \mathrm{~min})$ was similar to other groups [1, 9-12, 24]. Xue $\mathrm{H}$ et al. [9] compared the use of fluoroscopy-guided G-POEM vs conventional GPOEM procedure in 14 patients; all procedures achieved technical success, the PMR was identified in all 7 patients of the fluoroscopy-guided group, and only in 4(57.1\%) from the control $(P<0.03)$. However, this was not clinically expressed, with a non-statistically significant difference between GCSI and GES. In our group, LP provided a better orientation towards PMR identification and myotomy confirmation in all cases, including $5 / 28$ patients where the endoscopist was "lost", during tunnel creation and PMR was not identified, where the LP allowed the completion of G-POEM procedures, representing a $17.8 \%$ benefit in them. However, besides the fact that the G-POEM outcomes were slightly better in our study, when compared with other centers, with a general clinical success $(85.7 \%$ vs $69-81 \%)$ in GCSI and GES $(89.2 \%$ vs $69-84.2 \%)$ at 6 -month evaluations, we can't assume that this could be explained because of the $100 \%$ PMR identification (similar to the fluoroscopyguided group from Xue's study). However, as stated by other authors, different gastroparesis subtypes with their corresponding physiopathology could explain the real heterogeneous mid-term results more than the simple direct effect of the PMR cutting, inclusive with LP guidance, as in our patients $[1-4,9-11,24]$.

The strengths of our study include the use of LP in the two most common and important third-space procedures, the sample size that was reached in both and calculated for statistical significance, adequate and strict procedural and follow-up protocols, technical confirmation of all steps in all cases, and excellent safety without adverse events associated with LP use. Our study also has limitations that should be addressed. First, LP is not yet commercially available. Second, LP has to be made before each procedure by the medical doctor, which, in 
spite of the fact that it takes only between 10 and 15 min, could be time-consuming. Third, different LED and nasogastric feeding tube brands exist around the world, which limits the availability of the system we used. Fourth, only POEM and G-POEM cases were included; pediatric and other third-space procedures were not included, and fifth, LP was used in $76.2 \%$ of naïve POEM cases, which represent a subgroup of non-difficult cases in which LP could have been useless, especially when performed by highly-experienced endoscopists in thirdspace procedures; therefore, we think that the best advantage of LP use could be found in early-experience endoscopists in third-space procedures. However, we think that LP is a useful device for POEM and G-POEM procedures because of its simplicity, innovation, low costs, safety and the ability to make difficult procedures potentially easier.

\section{Conclusion}

In conclusion, we have confirmed the feasibility of using LP in third-space endoscopy as a new alternative to performing POEM or G-POEM cases, being specifically useful when classic anatomical landmarks are not completely reliable, in difficult cases, low-volume POEM and G-POEM centers, limited third-space procedures experience and when no other confirming methods are available. However, the real clinical relevance of LP use must be confirmed with longer evaluations and comparative studies. Commercialization and evaluation in other third-space procedures are necessary to elucidate potential advantages of the LP system.

\section{Supplementary information}

Supplementary information accompanies this paper at https://doi.org/10. 1186/s12876-020-01260-9.

Additional file 1: Video 1. Led Probe Construction. This is a video that we named led probe construction. In this video we show how to perform a LP before using in POEM or G-POEM cases.

Additional file 2: Video 2. LP clinical cases. In this video we show the use of LP in clinical POEM and G-POEM cases.

Additional file 3. POEM files. This is the POEM files from our cohort of patients with POEM that underwent to LP procedure.

Additional file 4. G-POEM files. This is the G-POEM files from our cohort of patients with G-POEM that underwent to LP procedure.

\section{Abbreviations}

LP: LED-probe; POEM: Peroral endoscopic myotomy; G-POEM: Gastric peroral endoscopic myotomy; GES: gastric emptying scintigraphy; IQR: interquartile range; IRP: Integrated relaxation pressure; EGJ: Esophagogastric junction; PMR: pyloric muscle ring; LED: Light-emitting diode; HRM: High resolution manometry; Upper GI: Upper gastrointestinal; CT: Computed tomography; TBE: Timed barium esophagogram; PPI: Proton pump inhibitor; GCSI: Gastroparesis Cardinal Symptom Index; RP4H: Retention Percentage $4 \mathrm{~h}$; OTSC: Over the scope clip; SD: Standard deviation

Acknowledgements

Not applicable

\section{Authors' contributions}

1.-OVHM: conception of LP protocol and idea, creation of manuscript, POEM and G-POEM cases, revision of final manuscript. 2.-RAZM: analysis and interpretation of the data, figures and tables creation. 3.-OMSP: acquisition of data and design, statistical analyses, POEM and G-POEM cases. 4.-RAGA: cases management and follow-up, analysis and interpretation of data. 5.-LFGC: POEM and G-POEM cases management and follow-up. All authors have read and approved the present manuscript

\section{Funding}

The authors declare not having any funding source for the development of the present study.

\section{Availability of data and materials}

All data generated or analyzed during this study are included in this published article [we added spv files as supplementary files].

\section{Ethics approval and consent to participate}

This protocol was approved by the Local Ethics Committee named: "Comité Local de Investigación y Ética en Investigación en Salud 3601 with registration number 13 Cl 09015184 before COFEPRIS" which belongs to "Hospital de especialidades Dr. Bernardo Sepúlveda Gutierrez, Centro Médico Nacional Siglo XXI del Instituto Mexicano del Seguro Social". They approved our protocol with the number: R-2016-3601-192; and registration number: 2016-CMN675). Written Informed consent was obtained from all patients.

\section{Consent for publication}

Not applicable.

\section{Competing interests}

OVHM, RAZM, OMSP, RAGA and LFGC, declare they do not have any competing interests.

Received: 28 November 2019 Accepted: 31 March 2020

Published online: 05 May 2020

\section{References}

1. Manolakis AC, Inoue H, Ueno A, Shimamura Y. 2007-2019: a "third"- space odyssey in the endoscopic Management of Gastrointestinal Tract Diseases. Curr Treat Options Gastroenterol. 2019;17:202-20.

2. Nabi Z, Nageshwar R, Ramchandani M. Recent advances in third-space endoscopy. Gastroenterol Hepatol. 2018;14:224-32.

3. Eleftheriadis N, Inoue H, Ikeda H, Onimaru M, Maselli R, Santi G. Submucosal tunnel endoscopy: Peroral endoscopic myotomy and peroral endoscopic tumor resection. World J Gastrointest Endosc. 2016;8:86-103.

4. Kobara H, Mori H, Rafiq K, MasaKI T. Submucosal tunneling techniques: current perspectives. Clin Exp Gastroenterol. 2014;5:67-74.

5. Li QL, Wu QN, Zhang XC, XU MD, Zhang W, Chen SY, Zhong YS, Zhang YQ, Chen WF, Qin WZ, Hu JW, Cai MY, Yao LQ, Zhou PH. Outcomes of per-oral endoscopic myotomy for treatment of esophageal achalasia with a median follow-up of 49 months. Gastrointest Endosc. 2018;87:1405-12.

6. Akintoye E, Kumar N, Obatian I, Alayo QA, Thompson CC. Peroral endoscopic myotomy: a meta-analysis. Endoscopy. 2016;48:1059-68.

7. Werner YB, Rösch T. POEM and submucosal tunneling. Curr Treat Options Gastroenterol. 2016:14:163-7.

8. Parikh MP, Gupta NM, Sanaka MR. Esophageal third space endoscopy: recent advances. Curr Treat Options Gastroenterol. 2019;17:63-75.

9. Xue HB, Fan HZ, Meng XM, Cristofaro S, Mekaroonkamol P, Dacha S, Li LY, Fu XL, Zhan SH, Cai Q. Fluoroscopy-guided gastric peroral endoscopic pyloromyotomy (G-POEM): a more reliable and efficient method for treatment of refractory gastroparesis. Surg Endosc. 2017;31:4617-24.

10. Shlomovitz E, Pescarus R, Cassera MA, Sharata AM, Reavis KM, Dunst CM, Swanstróm LL. Early human experience with per-oral endoscopic pyloromyotomy (POP). Surg Endosc. 2015;29:543-51.

11. Koul A, Dacha S, Mekaroonkamol P, Li X, Li L, Shahnavaz N, Keilin S, Willingham FF, Christie J, Cai Q. Fluoroscopic gastric peroral endoscopic myotomy (G-POEM) in patients with failed gastric electrical stimulator. Gastroenterol Rep. 2018;6:122-6.

12. Hong HJ, Song QW, Ko WJ, Jim MH, Hahm KB, Hong SP, Cho JY. Doublescope Peroral endoscopic Myotomy (POEM) for esophageal achalasia: the first trial of a new double-scope POEM. Clin Endosc. 2016;49:383-6. 
13. Grimes KL, Inoue H, Onimaru M, Ikeda H, Tansawet A, Bechara R, Tanaka S. Double-scope per oral endoscopic myotomy (POEM): a prospective randomized trial. Surg Endosc. 2016;30:1344-51.

14. Boyan WP Jr, Lavy D, Dinallo A, Otero J, Roding A, Hanos D, Dressner R, Arvanitis M. Lighted ureteral stents in laparoscopic colorectal surgery; a fiveyear experience. Ann Transl Med. 2017;5:44-8.

15. Hamilton AER, Westwood DA, Cuda TJ, Stevenson ARL. Identification of the ureter during robotic colorectal surgery using lighted stents - a video vignette. Color Dis. 2018;20:163-4.

16. Koelbl PS, Lingenfelder C, Spraul CW, Kampmeier J, Koch FH, Kim YK, _Hessling M. An intraocular micro light-emitting diode device for endoillumination during pars plana vitrectomy. Eur J Ophthalmol. 2019;29:75-81.

17. Pan JW, Su YC, Chen YS. Secondary optical element design for intracorporeal LED illumination system. Opt Lett. 2014;39:224-7.

18. Kahrilas PJ, Bredenoord AJ, Fox M, Gyawali CP, Roman S, Smout AJ, Pandolfino JE. The Chicago classification of esophageal motility disorders, v3.0. Neurogastroenterol Motil. 2015;27:160-74.

19. Rezende JM. Classificação radiológica do megaesôfago. Rev Goiana Med. 1982;28:187-91.

20. Eckardt VM, Aignherr C, Bernhard G. Predictors of outcome in patients with achalasia treated by pneumatic dilation. Gastroenterology. 1992;103:1732-8.

21. Inoue H, Minami H, Kobayashi Y, Sato Y, Kaga M, Suzuki M, Satodate H, Odaka N, Itoh H, Kudo S. Peroral endoscopic myotomy (POEM) for esophageal achalasia. Endoscopy. 2010;42:265-71.

22. Revicki DA, Rentz AM, Dubois D, Kahrilas P, Stangellini V, Talley NJ, Tack J. Development and validation of a patient-assessed gastroparesis symptom severity measure: the Gastroparesis cardinal symptom index. Aliment Pharmacol Ther. 2003;18:141-50.

23. Cotton PB, Eisen GM, Aabakken L, Baron TH, Hutter MM, Jacobson BC, Mergener K, Nemcek A Jr, Petersen BT, Petrini JL, Pike IM, Rabeneck L, Romagnuolo J, Vargo JJ. A lexicon for endoscopic adverse events: report of an ASGE workshop. Gastrointest Endosc. 2010;71:446-54.

24. Khashab MA, Steint E, Clarke JO, Saxena P, Kumbhari V, Chander Roland B, Kalloo AN, Stavropoulos S, Pasricha P, Inoue H. Gastric peroral endoscopic myotomy for refractory gastroparesis: first human endoscopic pyloromyotomy (with video). Gastrointest Endosc. 2013;78:764-8.

25. Sumiyama K, Gostout CJ, Rajan E, Bakken TA, Knipschield MA, Marler RJ. Submucosal endoscopy with mucosal flap safety valve. Gastrointest Endosc. 2007;65:688-94.

26. Grimes KL, Bechara R, Shimamura Y, Ikeda H, Inoue H. Gastric myotomy length affects severity but not rate of post-procedure reflux: 3-year followup of a prospective randomized controlled trial of double-scope per-oral endoscopic myotomy (POEM) for esophageal achalasia. Surg Endosc Aug. 2019;28. https://doi.org/10.1007/s00464-019-07079-0.

\section{Publisher's Note}

Springer Nature remains neutral with regard to jurisdictional claims in published maps and institutional affiliations.

Ready to submit your research? Choose BMC and benefit from:

- fast, convenient online submission

- thorough peer review by experienced researchers in your field

- rapid publication on acceptance

- support for research data, including large and complex data types

- gold Open Access which fosters wider collaboration and increased citations

- maximum visibility for your research: over $100 \mathrm{M}$ website views per year

At $\mathrm{BMC}$, research is always in progress.

Learn more biomedcentral.com/submissions 